\title{
Dose Sparing Potential of Deep Inspiration Breath-hold Technique for Left Breast Cancer Radiotherapy Organs-at-risk
}

\author{
GAVIN LAWLER $^{1,2}$ and MICHELLE LEECH ${ }^{2}$ \\ ${ }^{1}$ Beacon Hospital, Sandyford, Dublin, Ireland; \\ ${ }^{2}$ Discipline of Radiation Therapy, Trinity College, Dublin, Ireland
}

\begin{abstract}
Background/Aim: To assess if deep inspiration breath-hold (DIBH) technique achieved dose sparing for organs-at-risk in left breast radiotherapy patients in order to reduce long-term complications. Patients and Methods: DIBH and Free-breathing $(F B)$ as a control, CT planning scans obtained for 28 left breast/chest wall (+/supraclavicular field) patients treated January 2008December 2013 were retrospectively re-contoured and replanned. Organs-at-risk examined: lungs, left lung, heart and left anterior descending coronary artery (LADCA). Quantitative statistical analysis of plan dose differences was performed. Results: Lung dose was not affected by DIBH. Heart $D_{\text {max }}$ reduced by $34.5 \%(F B=41.81 G y, S D=3.963 G y$ vs. $D I B H=27.39 G y, S D=12.393 G y, p<0.000004)$. Heart $D_{\text {mean }}$ reduced by $32.6 \%(F B=1.817 G y, S D=0.627 G y$ vs. $D I B H=1.224 G y, S D=0.344 G y, p=0.00000083067 . L A D C A$ $D_{\max }$ reduced by $47.8 \%$ (DIBH mean $=15.56 G y$, $S D=10.62 G y$ vs. $F B$ mean $=29.82 G y, \quad S D=10.05 G y$, $p=0.000031$, and LADCA $D_{\text {mean }}$ by $52 \% \quad(D I B H$ mean $=5.23 G y, \quad S D=1.94 G y$ vs. $F B$ mean $=10.88 G y$, $S D=3.95 G y p=0.000000036027$. Amplitude depths were not correlated with dose reductions. Conclusion: DIBH significantly reduces heart and LADCA dose. Further research is required to evaluate potential long-term implications for patients treated DIBH.
\end{abstract}

Breast cancer accounts for over $20 \%$ of all cancer diagnoses internationally, being the most prevalent gynecological cancer following non-melanoma skin cancer (1). Optimal treatment and management is essential. Radiotherapy plays

Correspondence to: Gavin Lawler, Research Office Radiotherapy Department, Beacon Hospital, Sandyford, Dublin 18, Ireland. Personal Address: Gavin Lawler, 11 Greenhills, Athy, Co.Kildare, Ireland. Tel: +353 12936691, Fax: +353 12936657, e-mail: lawlerg@tcd.ie

Key Words: Breast cancer, radiotherapy, deep inspiration breathhold, cardiac dose. a key role in breast cancer management (2). Adjuvant radiotherapy has been shown to increase survival rates and reduce loco-regional recurrences $(3,4)$. Fisher et al. highlighted that radiotherapy, when used after breastconservative surgery can reduce patients' local recurrence risk by over $60 \%$. Survival rates were also found to equal those of mastectomy patients (5). However, while radiotherapy effectively reduced cancer mortality, chronic long-term side-effect complications affected patients' overall mortality and survival rates. Even though cancer mortality was decreasing, patients were dying as a result of treatment side-effects (6).

Cardiac complications such as myocardial infarction, congestive heart failure, coronary artery disease and ischaemia presented long-term post-radiotherapy (6). Pulmonary complications post-radiotherapy have also been investigated. Radiation pneumonitis has routinely been reported in 1-5\% of patients and lung fibroses have even been documented (earlier techniques with excessive lung volumes in treatment fields) (7). Gagliardi et al. used the relative seriality model to analyze the complication probability of radiation pneumonitis (8). A strong dose volume effect was established for pneumonitis risk. It is important therefore to consider the dose volume relationship and mean lung dose, in order to quantify potential pneumonitis risk.

Contrastingly, evidence is conflicting regarding specific heart anatomy most vulnerable to inducing cardiac complications post-radiotherapy. Focus mainly considers induction of coronary artery disease, perfusion defects, pericarditis and ischaemic events as a result of coronary arterial dose, ventricular dose, the volume of heart irradiated, and patient-specific factors that influence increased cardiac dose on treatment.

With conventional tangential radiotherapy the volume of heart irradiated and heart volume within the treatment field may influence potential cardiac risk. The heart, left ventricle and pericardium may potentially receive doses from the technique used to deliver treatment. The coronary arteries may receive dose as a result of field arrangement 
and scatter effects, particularly given their anatomical location. They can be difficult to distinguish/contour accurately on current CT planning systems and further imaging techniques have been recommended such as IV contrast, magnetic resonance imaging and single positron emission computer tomography to achieve accurate delineation for examination of the dose they receive. Feng et al. developed a specific radiation oncology cardiac atlas to aid delineation of cardiac substructures (with and without IV contrast) to reduce contouring discrepancies and attempt standardization (9).

Until a direct link is established, it is imperative that current treatment techniques and methods such as DIBH, are investigated to minimize cardiac and pulmonary doses where it is possible, without compromising therapeutic dose.

\section{Patients and Methods}

Study design. Ethical approval was sought and granted from Trinity College Dublin and Beacon Hospital Research Ethics Committee. The study comprised of a retrospective historically treated patient cohort that received left breast/left chest wall +/- left supraclavicular radiotherapy between January 2008 and December 2013 as part of their cancer management.

Participant population. 56 treatment plans were created for 28 patients and each patient acted as their own control. Inclusion criteria dictated patients were female, aged 18 years or older and able to give informed consent, with a diagnosis of breast cancer that required left breast or left chest wall plus or minus left supraclavicular radiotherapy treatment. Both a free breathing and DIBH CT planning scan were also required for comparative purposes, hence patients must have been able to perform and maintain DIBH for a minimum of ten seconds, in order to acquire DIBH CT planning scan.

Data collection. Patients were immobilized supine via a breast-board or wing-board and vacuum bag if treating supraclavicularn area, both arms up and knee-fix. A 16 head GE Lightspeed CT scanner using $2.5 \mathrm{~mm}$ slice thickness was used and breathing was monitored using Varian Real-time Position Management (RPM) respiration synchronized imaging and treatment system versions 1.7-1.7.5.

Contouring. CT scans were contoured and planned by the lead investigator using Varian Eclipse Treatment Planning Software Version 11.031. Contours included the combined lungs, left lung, heart and LADCA. Feng et al.'s cardiac atlas guidelines and RTOG Breast Atlas Contours were followed $(9,10)$.

The lungs were auto-contoured using Eclipse wizard software and manually corrected accordingly on lung windows. The heart was contoured superiorly from below the arch of the aorta down to include all visible pericardium inferiorly. Contoured heart volumes agreed within 50cc between FB and DIBH scans. The LADCA was contoured superiorly extending from the left coronary artery and inferiorly down the interventricular groove adjacent to the left and right ventricles. The windowing level required significant adjustment to contour the LADCA, particularly in the heart apex at
Table I. Combined lungs dose (D5\%-D90\%) free breathing (FB) versus deep inspiration breath-hold (DIBH).

\begin{tabular}{lccccc}
\hline D\% & FB & SD & DIBH & SD & $p$-Value \\
\hline 5 & 28.886 & 13.368 & 24.111 & 12.718 & 0.052 \\
10 & 10.124 & 10.013 & 8.147 & 6.612 & 0.134 \\
20 & 3.262 & 5.342 & 2.445 & 1.263 & 0.336 \\
30 & 1.205 & 0.830 & 1.156 & 0.482 & 0.627 \\
40 & 0.659 & 0.264 & 0.689 & 0.220 & 0.400 \\
50 & 0.244 & 0.127 & 0.279 & 0.120 & 0.085 \\
60 & 0.147 & 0.132 & 0.136 & 0.075 & 0.660 \\
70 & 0.082 & 0.112 & 0.067 & 0.060 & 0.470 \\
80 & 0.026 & 0.044 & 0.028 & 0.043 & 0.607 \\
90 & 0.014 & 0.030 & 0.009 & 0.022 & 0.120 \\
\hline
\end{tabular}

$\mathrm{SD}$, Standard deviation; All figures have been rounded to 3 decimals.

the inferior extent.

Treatment planning. 6MV field-in-field technique was used for all plans, with low weighted $15 \mathrm{MV}$ beams occasionally used as required. Supraclavicular field plans were mono-isocentric. Tangential field posterior borders were matched to reduce internal divergence, with no boost volumes planned. Departmental planning constraints used and adhered to were as follows:

- Heart: $\mathrm{V}_{40 \mathrm{~Gy}} \leq 5 \%, \mathrm{~V}_{20 \mathrm{~Gy}} \leq 10 \%, \mathrm{D}_{\max }<50$ Gy

- Lung: $\mathrm{V}_{20 \mathrm{~Gy}} \leq 20 \%$.

The LADCA was not considered in plan optimization but dose to this structure was reported in all cases.

ICRU dose constraints were met for all treatment plans, with one patient exception (111.1\% free-breathing (FB), 110\% DIBH Plan Maximum) (11). Plans were calculated using AAA calculation algorithm version 8.9.17 and prescribed between 40.05Gy-50Gy in 1525 fractions respectively. Cardiac shielding (without compromising target dose) was performed on all plans. This ensured a true comparison between FB and DIBH plans, eliminating potential variance.

Data analysis. IBM SPSS Statistics Version 21.0 was used for data analysis. For each organ, the specific dose to each volume, $\mathrm{D}_{10 \%}$ to $\mathrm{D}_{100 \%}$ inclusive, was investigated to determine dose impact of DIBH versus FB. For comparative purposes, maximum dose $\left(\mathrm{D}_{\max }\right)$, mean dose $\left(D_{\text {mean }}\right), V_{30 G y}$ and $V_{20 G y}$ were also assessed (12-17). Paired student sample $t$-tests were used, examining dose differences between FB and DIBH plans for organs-at-risk. Pearson correlations were performed to assess relationships between variables. Differences in amplitude level and the dose impact were examined using independent sample t-tests. With multiple $t$-tests performed (75) a Bon Ferroni correction was carried out, to reduce potential statistical errors. The corrected significance level was $p<0.00066667$.

\section{Results}

Demographics. Patients' age ranged between 37 and 75 years, with mean age being 57.39 years, $\mathrm{SD}=8.412$. The majority of patients were between 50 and 60 years old. 
Table II. Left breast radiotherapy organs-at-risk $D_{\text {max }} \& D_{\text {mean }}$ free breathing $(F B)$ versus DIBH.

\begin{tabular}{|c|c|c|c|c|c|c|}
\hline Organ measurement & Technique & Mean Dose (Gy) & $\mathrm{SD}$ & Mean Difference (Gy) & SD & $p$-Value \\
\hline \multirow{2}{*}{ Lungs $D_{\max }$} & FB & 48.329 & 3.447 & -0.041 & 1.786 & 0.905 \\
\hline & DIBH & 48.369 & 3.125 & & & \\
\hline \multirow[t]{2}{*}{ Lungs $\mathrm{D}_{\text {mean }}$} & FB & 3.743 & 1.787 & 0.356 & 1.138 & 0.110 \\
\hline & DIBH & 3.387 & 1.158 & & & \\
\hline \multirow[t]{2}{*}{ Left lung $D_{\max }$} & FB & 48.329 & 3.447 & -0.104 & 1.748 & 0.756 \\
\hline & DIBH & 48.432 & 3.064 & & & \\
\hline \multirow[t]{2}{*}{ Left lung $D_{\text {mean }}$} & FB & 8.150 & 3.743 & 0.989 & 2.489 & 0.045 \\
\hline & DIBH & 7.160 & 2.379 & & & \\
\hline \multirow{2}{*}{ Heart $D_{\max }$} & FB & 41.810 & 3.963 & 14.421 & 13.347 & 0.000 \\
\hline & DIBH & 27.389 & 12.393 & & & \\
\hline \multirow[t]{2}{*}{ Heart $D_{\text {mean }}$} & FB & 1.817 & 0.627 & 0.593 & 0.493 & 0.000 \\
\hline & DIBH & 1.224 & 0.344 & & & \\
\hline \multirow{2}{*}{ LADCA $D_{\max }$} & FB & 29.820 & 10.051 & 14.266 & 15.131 & 0.000 \\
\hline & DIBH & 15.555 & 10.615 & & & \\
\hline \multirow[t]{2}{*}{ LADCA $D_{\text {mean }}$} & FB & 10.884 & 3.953 & 5.658 & 3.942 & 0.000 \\
\hline & DIBH & 5.226 & 1.943 & & & \\
\hline
\end{tabular}

SD, Standard deviation; Mean Difference, dose difference between techniques. All figures have been rounded to 3 decimals.

Table III. Free breathing (FB) versus DIBH $V_{30 G y}$ and $V_{20 G y}$ for the combined lungs \& heart.

\begin{tabular}{|c|c|c|c|c|c|c|c|}
\hline Organ & Technique & Mean volume $\%$ & SD & Mean difference volume $\%$ & SD & $p$-Value & Mean volume reduction $\%$ \\
\hline \multirow{2}{*}{ Lungs $V_{30 G y}$} & FB & 5.62 & 3.95 & 0.72 & 2.78 & 0.184 & 12.7 \\
\hline & DIBH & 4.91 & 2.23 & & & & \\
\hline \multirow[t]{2}{*}{ Lungs $V_{20 G y}$} & FB & 6.61 & 4.49 & 0.83 & 3.28 & 0.191 & 12.6 \\
\hline & DIBH & 5.78 & 2.55 & & & & \\
\hline \multirow[t]{2}{*}{ Heart $V_{30 G y}$} & FB & 0.47 & 1.03 & 0.43 & 1.06 & 0.043 & 90.4 \\
\hline & DIBH & 0.05 & 0.18 & & & & \\
\hline \multirow[t]{2}{*}{ Heart $V_{20 G y}$} & FB & 0.89 & 1.18 & 0.76 & 1.24 & 0.003 & 86.2 \\
\hline & DIBH & 0.12 & 0.32 & & & & \\
\hline
\end{tabular}

SD, Standard deviation; Mean Volume Reduction\%, mean difference between FB \& DIBH. All volumes have been rounded to 2 decimals.

Invasive ductal carcinoma accounted for $64.3 \%, n=18$ patients' diagnoses and a further $14.3 \%, \mathrm{n}=4$ patients' with ductal carcinoma in situ (DCIS). Invasive lobular carcinoma and inflammatory breast cancer each accounted for $3.6 \%, n=1$ patient's diagnosis, respectively. None of the sample had metastatic disease, however $25 \%, \mathrm{n}=7$ patients had 1 involved node and $14.3 \%, n=4$ patients required supraclavicular irradiation. Grade 2 disease was reported in $60.7 \%, \mathrm{n}=17$ patients staging, while $21.4 \%, \mathrm{n}=6$ were Grade 3. Wide local excision had been performed on $89.3 \%, \mathrm{n}=25$ patients, ipsilateral mastectomy on $7.1 \%, \mathrm{n}=2$ and bilateral mastectomy on $3.6 \%, \mathrm{n}=1$ patient. Chemotherapy was given to $67.9 \%, \mathrm{n}=19$ patients, whilst $14.3 \%, \mathrm{n}=4$ patients had previously treated right breast cancer. Co-morbidities were reported by $57.1 \%, \mathrm{n}=16$ patients, however none reported any lung issues. Preexisting hypertension was reported in $17.9 \%, \mathrm{n}=5$ patients and angina in $3.6 \%, \mathrm{n}=1$ patient.
The combined lungs. Participants' lung volumes, significantly increased with DIBH (mean=4632.52 $\mathrm{cm}^{3}, \mathrm{SD}=796.13 \mathrm{~cm}^{3}$ ) compared to FB (mean=2866.61 $\left.\mathrm{cm}^{3}, \mathrm{SD}=602.51 \mathrm{~cm}^{3}\right)$, $\mathrm{t}(27)=-17.26, p=0.00000000000000021212$. No significant dose differences were noted for any lung volumes regardless of technique (Table I). $\mathrm{D}_{\text {mean }}$ was not affected by DIBH (mean=3.39 Gy, SD=1.16 Gy vs. FB mean=3.74 Gy, SD=1.79 Gy, $\mathrm{t}(27)=1.652, \quad p=0.110)$. $\mathrm{D}_{\max }$ was also comparable (FB mean=48.33 Gy, SD=3.45 Gy vs. DIBH mean=48.37 Gy, $\mathrm{SD}=3.12 \mathrm{~Gy}), \mathrm{t}(27)=-0.121, p=0.905$, Table II). No improvement was observed for $\mathrm{V}_{30 \mathrm{~Gy}}$ (DIBH mean $=4.91 \%, \mathrm{SD}=2.23 \%$ vs. FB mean $=5.62 \%, \mathrm{SD}=3.95 \%), \mathrm{t}(27)=1.363, p=0.184 . \mathrm{V}_{20 \mathrm{~Gy}}$ was similar $(\mathrm{DIBH}$ mean $=5.78 \%, \mathrm{SD}=2.55 \%$ vs. $\mathrm{FB}$ mean $=6.61 \%$, $\mathrm{SD}=4.49 \%, \mathrm{t}(27)=1.34, p=0.191$, Table III).

Ipsi-lateral lung dose. Unsurprisingly, left lung results reflected the combined lungs' evaluation with no notable dose differences observed between FB and DIBH (Table IV). 
Table IV. Left lung dose $\left(D_{5 \%}-D_{100 \%}\right.$ free breathing $(F B)$ versus DIBH

\begin{tabular}{lccccc}
\hline D\% & FB & SD & DIBH & SD & $p$-Value \\
\hline 5 & 39.432 & 10.111 & 39.750 & 6.279 & 0.791 \\
10 & 31.355 & 12.744 & 26.011 & 12.390 & 0.027 \\
20 & 11.782 & 10.217 & 9.315 & 7.291 & 0.080 \\
30 & 6.103 & 7.810 & 4.500 & 2.883 & 0.162 \\
40 & 3.796 & 5.981 & 2.753 & 1.418 & 0.277 \\
50 & 2.334 & 2.800 & 1.871 & 0.919 & 0.268 \\
60 & 1.504 & 1.221 & 3.411 & 11.094 & 0.368 \\
70 & 1.064 & 0.609 & 0.987 & 0.391 & 0.320 \\
80 & 0.822 & 0.341 & 0.779 & 0.264 & 0.403 \\
90 & 0.641 & 0.216 & 0.587 & 0.205 & 0.181 \\
100 & 0.153 & 0.123 & 0.056 & 0.080 & 0.000 \\
\hline
\end{tabular}

$\mathrm{SD}$, Standard deviation; All figures have been rounded to 3 decimals.

The left lung's volume expansion increased significantly with DIBH $\left(\right.$ mean $\left.=2153.02 \mathrm{~cm}^{3}, \mathrm{SD}=414.62 \mathrm{~cm}^{3}\right) v s$. FB (mean=1293.98 $\mathrm{cm}^{3}, \quad \mathrm{SD}=323.27 \quad \mathrm{~cm}^{3}$ ), mean difference $=859.04 \mathrm{~cm}^{3}, \mathrm{SD}=258.21 \mathrm{~cm}^{3}, \mathrm{t}(27)=-17.605$, $p=0.00000000000000025169$ ).

Left lung $\mathrm{D}_{\text {mean }}$ was similar regardless $(\mathrm{FB}$ mean $=8.15$ $\mathrm{Gy}, \mathrm{SD}=3.74 \mathrm{~Gy} v s$. DIBH mean=7.16 Gy, $\mathrm{SD}=2.38 \mathrm{~Gy}$, mean difference $=0.99 \mathrm{~Gy}, \mathrm{SD}=2.19 \mathrm{~Gy}, \mathrm{t}(27)=2.104$, $p=0.045$, Table II).

The heart. Cardiac dose was reduced for all volumes using DIBH (significant reductions between $\mathrm{D}_{10 \%}-\mathrm{D}_{50 \%}$ inclusive, Table V). Heart $\mathrm{D}_{\max }$ reduced $34.5 \%$ (FB mean $=41.81 \mathrm{~Gy}$, $\mathrm{SD}=3.963$ Gy $v s$. DIBH mean=27.39 Gy, SD=12.393 Gy, mean difference $=14.421 \mathrm{~Gy}, \mathrm{SD}=13.347 \mathrm{~Gy}, \mathrm{t}(27)=5.717$, $p=0.00000083067$ Table II). $\mathrm{D}_{\text {mean }}$ considerably reduced using DIBH (mean $=1.224 \mathrm{~Gy}, \mathrm{SD}=0.344 \mathrm{~Gy}$ ) versus $\mathrm{FB}$ (mean $=1.817 \mathrm{~Gy}, \mathrm{SD}=0.627 \mathrm{~Gy}$ ) at $32.6 \%$, mean difference $=0.593 \mathrm{~Gy}, \mathrm{SD}=0.493 \mathrm{~Gy}, \mathrm{t}(27)=6.357, p<0.000$ (Table II). A $90.4 \%$ volume reduction for heart $\mathrm{V}_{30 \mathrm{~Gy}}$ was not significant between $\mathrm{FB}$ (mean $=0.47 \%, \mathrm{SD}=1.03 \%$ ) and DIBH (mean $=0.045 \%, \quad \mathrm{SD}=0.177 \%)$, mean volume difference $=0.43 \%, \mathrm{SD}=1.058 \%, \mathrm{t}(27)=2.128, p=0.043$. Heart $\mathrm{V}_{20 \mathrm{~Gy}}$ experienced an $86.2 \%$ notable reduction using DIBH (mean $=0.122 \%, \mathrm{SD}=0.321 \%) \quad v s . \quad \mathrm{FB} \quad$ (mean $=0.885 \%$, $\mathrm{SD}=1.182 \%)$, mean difference $=0.763 \%, \mathrm{SD}=1.24 \%$, $\mathrm{t}(27)=3.263, p=0.003$. However, this was not significant, Table III. DIBH removed heart in the field for $28.6 \%$, $(n=8$ participants). Overall, a $55.9 \%$ reduction in mean heart volume within the beam's eye view was observed.

Left Anterior Descending Coronary Artery (LADCA). The LADCA experienced significant dose reductions for all volumes examined using DIBH, Table VI. LADCA $\mathrm{D}_{\max }$ reduced by $47.83 \%$ using $\mathrm{DIBH}$ (mean=15.56Gy, $\mathrm{SD}=10.62 \mathrm{~Gy}) v s$. FB
Table V. Heart dose $\left(D_{5 \%}-D_{100 \%}\right)$ treated free breathing $(F B)$ versus $D I B H$.

\begin{tabular}{lcccccc}
\hline D\% & FB & SD & DIBH & SD & $p$-Value & $\%$ reduction \\
\hline 5 & 6.710 & 5.530 & 3.548 & 1.136 & 0.003 & $47 \%$ \\
10 & 3.435 & 1.177 & 2.489 & 0.685 & 0.000 & $27 \%$ \\
15 & 2.508 & 0.604 & 1.982 & 0.500 & 0.000 & $21 \%$ \\
20 & 2.051 & 0.461 & 1.667 & 0.406 & 0.000 & $18.7 \%$ \\
30 & 1.501 & 0.326 & 1.254 & 0.297 & 0.000 & $16.5 \%$ \\
40 & 1.148 & 0.256 & 0.984 & 0.241 & 0.000 & $14.4 \%$ \\
50 & 0.894 & 0.205 & 0.781 & 0.197 & 0.000 & $12.7 \%$ \\
60 & 0.697 & 0.167 & 0.622 & 0.164 & 0.004 & $10.6 \%$ \\
70 & 0.541 & 0.134 & 0.490 & 0.149 & 0.021 & $9.4 \%$ \\
80 & 0.414 & 0.106 & 0.368 & 0.125 & 0.121 & $6.8 \%$ \\
90 & 0.304 & 0.083 & 0.297 & 0.097 & 0.643 & $2.2 \%$ \\
100 & 0.127 & 0.075 & 0.118 & 0.068 & 0.519 & $7.2 \%$ \\
\hline
\end{tabular}

FB, Mean dose (Gy); DIBH, mean dose (Gy); SD, standard deviation; $\%$ reduction, relative reduction between FB and DIBH. All doses have been rounded to 3 decimals.

Table VI. LADCA dose $\left(D_{10 \%}-D_{100 \%}\right)$ treated free breathing $(F B)$ versus $D I B H$.

\begin{tabular}{lccccc}
\hline $\mathrm{D} \%$ & FB & SD & DIBH & SD & \% Reduction \\
\hline 10 & 19.43 & 9.40 & 8.37 & 5.62 & 56.9 \\
20 & 16.25 & 8.13 & 6.24 & 2.02 & 61.6 \\
30 & 13.87 & 7.08 & 5.58 & 1.62 & 59.8 \\
40 & 11.5 & 4.47 & 5.12 & 1.44 & 55.5 \\
50 & 9.65 & 3.23 & 4.74 & 1.33 & 50.9 \\
60 & 8.01 & 2.92 & 4.39 & 1.26 & 45.2 \\
70 & 6.70 & 2.73 & 4.05 & 1.2 & 39.5 \\
80 & 5.59 & 2.40 & 3.69 & 1.13 & 34 \\
90 & 4.45 & 1.78 & 3.28 & 1.08 & 27.8 \\
100 & 2.83 & 0.95 & 2.05 & 0.72 & 27.4 \\
\hline
\end{tabular}

SD, Standard deviation; \% Reduction, dose reduction between FB and DIBH. All doses have been rounded to 2 decimals.

(mean=29.82 Gy, SD=10.05 Gy), mean difference=14.27 Gy, $\mathrm{SD}=15.13 \mathrm{~Gy}, \mathrm{t}(27)=4.989, p=0.000031$. LADCA $\mathrm{D}_{\text {mean }}$ reduced using DIBH (mean=5.23 Gy, $\mathrm{SD}=1.94$ Gy vs. $\mathrm{FB}$ mean $=10.88 \mathrm{~Gy}, \mathrm{SD}=3.95 \mathrm{~Gy}$, mean difference $=5.66 \mathrm{~Gy}$, $\mathrm{SD}=3.94 \mathrm{~Gy}, \mathrm{t}(27)=7.595, p=0.000000036027)$, giving a $52 \%$ reduction, Table II. Overall, DIBH proved beneficial to reduce LADCA dose.

Amplitude. Participants' amplitude, defined as the measurement from baseline expiration to their breath-hold, ranged from $0.6 \mathrm{~cm}-2.8 \mathrm{~cm}$. The most frequent sample amplitude was $2.0 \mathrm{~cm}$, obtained by $14.3 \%, \quad(\mathrm{n}=4)$ participants. The sample mean amplitude was $1.614 \mathrm{~cm}$, $\mathrm{SD}=0.55 \mathrm{~cm} .50 \%$ of $(\mathrm{n}=14)$ participants had amplitudes 
Table VII. The effect of amplitude level (high versus low) on left breast radiotherapy organs-at-risk $D_{\text {max }}$ and $D_{\text {mean }}$ treated DIBH.

\begin{tabular}{|c|c|c|c|c|c|}
\hline Measurement & Amplitude category & Mean dose Gy & SD & Mean difference Gy & $p$-Value \\
\hline \multirow[t]{2}{*}{ Lungs $D_{\max }$} & High & 48.77 & 2.58 & 0.80 & 0.506 \\
\hline & Low & 47.97 & 3.65 & & \\
\hline \multirow[t]{2}{*}{ Lungs $\mathrm{D}_{\text {mean }}$} & High & 3.22 & 0.79 & -0.33 & 0.468 \\
\hline & Low & 3.55 & 1.45 & & \\
\hline \multirow[t]{2}{*}{ Left lung $D_{\max }$} & High & 48.87 & 2.43 & 0.88 & 0.460 \\
\hline & Low & 48.00 & 3.63 & & \\
\hline \multirow[t]{2}{*}{ Left lung $D_{\text {mean }}$} & High & 6.84 & 1.64 & -0.64 & 0.491 \\
\hline & Low & 7.48 & 2.97 & & \\
\hline \multirow[t]{2}{*}{ Heart $D_{\max }$} & High & 28.09 & 12.18 & 1.40 & 0.772 \\
\hline & Low & 26.69 & 13.03 & & \\
\hline \multirow[t]{2}{*}{ Heart $D_{\text {mean }}$} & High & 1.20 & 0.44 & -0.05 & 0.713 \\
\hline & Low & 1.25 & 0.22 & & \\
\hline \multirow[t]{2}{*}{ LADCA $D_{\max }$} & High & 15.72 & 11.02 & 0.32 & 0.938 \\
\hline & Low & 15.39 & 10.60 & & \\
\hline \multirow[t]{2}{*}{ LADCA $_{\text {mean }}$} & High & 5.25 & 2.36 & 0.04 & 0.960 \\
\hline & Low & 5.21 & 1.51 & & \\
\hline
\end{tabular}

SD, Standard deviation; Mean Difference, difference between high and low. All doses have been rounded to 2 decimals.

above $1.614 \mathrm{~cm}$ and $50 \%(\mathrm{n}=14)$ below it, dividing the sample into high $(>1.6 \mathrm{~cm})$ and low $(<1.6 \mathrm{~cm})$ categories. Whether participants had a high or low amplitude was not found to be statistically relevant, amplitude dose effect was negligible for organs-at-risk, Table VII. Age and amplitude were established as having a significant relationship, such that as the participants' age increased their amplitude decreased, $\mathrm{r}(26)=-0.474, p=0.011$. However, while the relationship was significant its' negative basis was not.

Lung volume expansion difference and amplitude had no detectable relationship, $\mathrm{r}(26)=0.199, p=0.311$. Amplitude depth was not impacted by participants' smoking history, $\mathrm{r}(26)=-0.245, p=0.209$. Coincidentally, smoking history also had no relationship with lung expansion obtained between FB and DIBH, $\mathrm{r}(26)=0.048, p=0.808$. Suffering from comorbidities was the only other variable, that had a relationship with amplitude, $\mathrm{r}(26)=0.416, p=0.028$. Overall, amplitude level did not result in any significant impact for participants in relation to affecting dose received or volumes irradiated.

\section{Discussion}

The Combined Lungs \& Ipsi-lateral Lung

Participants' overall lung volumes increased significantly with DIBH as expected. Minimal dose differences were noted for each technique, Tables III, IV. However, regardless of technique used the doses were statistically comparable. Combined lungs $\mathrm{D}_{\text {mean }}$ and left lung $\mathrm{D}_{\text {mean }}$ were comparable between techniques. Marks et al. highlighted mean lung dose as an important factor for induction of radiation pneumonitis (18). To limit radiation pneumonitis risk to $\leq 20 \%$ Marks et al recommend using: $\mathrm{V}_{20 \mathrm{~Gy}} \leq 30-35 \%$ and mean lung dose
$\mathrm{D}_{\text {mean }} \leq 20-23 \mathrm{~Gy}$. The lungs had a mean dose range of 0.43 $5.0 \mathrm{~Gy}$ for DIBH with an overall mean dose, $\mathrm{D}_{\text {mean }}=3.39 \mathrm{~Gy}$, equating to reductions ranging between $83-85 \%$ less than Marks et al.'s suggested clinical data for $\leq 20 \%$ risk of radiation pneumonitis induction. Even the highest value for $\mathrm{DIBH}, \mathrm{D}_{30 \%}=5 \mathrm{~Gy}$, was $75-78.3 \%$ less than Marks' recommended level, ensuring participants within this study have a reduced risk of radiation pneumonitis. However, the mean lung dose was not improved enough between FB and $\mathrm{DIBH}$ to further reduce this risk. Correspondingly for $\mathrm{V}_{30 \mathrm{~Gy}}$ and $\mathrm{V}_{20 \mathrm{~Gy}}$ no significant advantage or disadvantage for the lungs was detected using DIBH versus FB, Table III.

The lungs have a strong dose-volume relationship to quantify potential complication risk. They can recover from small sections irradiated to high doses however, large volumes receiving dose compromises function and complications may result, highlighting their parallel organat-risk architecture (8). This may aid interpretation of results explaining why DIBH had no impact over FB. While a larger lung portion is potentially irradiated using DIBH, with lung expansion (significantly increased on breath-hold), there is less actual lung tissue within the irradiated volume resulting in similar lung dose overall.

Hayden et al. published similar results using DIBH (16). In contrast Swanson et al., concluded that lung doses were reduced with moderate DIBH, particularly the left lung mean dose (9.08 Gy vs. $7.86 \mathrm{~Gy}, p<0.001)$, a relative reduction of $13 \%$ observed over FB treatment (13).

Hayden et al. also used the RPM system, while Swanson et al. used active breathing control (ABC) which may account for result differences. ABC can influence patients' lung volume expansion and diaphragm placement compared 
to RPM's external surrogate tracking system. RPM does not impact on patients' natural breathing cycle; be it diaphragm or chest wall based for motion. ABC impacts on patients' breathing cycle and this could possibly influence the amount of lung tissue irradiated, causing hyperinflation, resulting in the reduction reported by Swanson et al. The lungs' expansion with DIBH compensates for the potentially increased lung volume irradiated, as the irradiated tissue quantity overall is reduced by expansion. Consequently, comparable results for FB and DIBH treatments occur.

DIBH positively reduced heart dose, Table V. While $\mathrm{D}_{5 \%}$ reduced by $47 \%$, this was not significant. $\mathrm{D}_{10 \%}-\mathrm{D}_{50 \%}$ inclusive, received significant dose reductions ranging between 12.7-27\%. Cardiac dose sparing from DIBH, could potentially lead to long-term reductions in cardiac complications. Heart $\mathrm{D}_{\max }$ benefitted by reducing $34.5 \%$ with DIBH (FB mean=41.81 Gy, SD=3.963 Gy vs. DIBH mean=27.39 Gy, $\mathrm{SD}=12.393 \mathrm{~Gy}, p<0.000$, Table II). Bruzzaniti et al. reported a superior $\mathrm{D}_{\max }$ reduction of $78 \%$ (19). However, full cardiac shielding was not specified in their treatment plans as was here, hence this may account for notable result discrepancy.

Heart $\mathrm{D}_{\text {mean }}$ was significantly reduced by $32.6 \%$ (FB mean=1.817 Gy, SD=0.627 Gy vs. DIBH mean dose=1.224 Gy, $\mathrm{SD}=0.344 \mathrm{~Gy}, p<0.000$, Table II). This is below Hayden et al., Swanson et al. and Nissen et al.'s reported reductions of $43.5 \%$, $40 \%$ and $48 \%$ respectively $(16,13,15)$. Again, cardiac shielding was not specified within these studies, which may account for the discrepancy. Considering Darby et al. established a linear $7.4 \%$ increase in major cardiac events for every $1 \mathrm{~Gy}$ increase in breast patients' heart $\mathrm{D}_{\text {mean }}$, this is potentially important (20). A $1 G y$ reduction in heart $D_{\text {mean }}$ could theoretically be clinically beneficial in reducing cardiac complications. Here, a mean difference of $0.593 \mathrm{~Gy}$ was achieved between techniques which could impact reduction of major cardiac events, in line with Darby et al.

Heart $\mathrm{V}_{30 \mathrm{~Gy}}$ reduced by $90.4 \%$, however this was not significant $(\mathrm{FB}$ mean $=0.47 \%, \mathrm{SD}=1.03 \%$ vs. $\mathrm{DIBH}$ mean volume $=0.045 \%, \mathrm{SD}=0.177 \%, p=0.043)$. This contrasts with Swanson et al.'s significant $88 \%$ reduction (FB $3.2 \%$ vs. DIBH $0.39 \%, p<0.001)$ and Hayden et al.'s $66 \%$ reduction, (FB 7.1\% vs. DIBH 2.4\%, $p<0.0001)(13,16)$. Equally, heart $\mathrm{V}_{20 \mathrm{~Gy}}$ also experienced a sizable $86.2 \%$ reduction (FB mean $=0.885 \%, \mathrm{SD}=1.182 \%$ vs. $\mathrm{DIBH}$ mean $=0.122 \%$, $\mathrm{SD}=0.321 \%, p=0.003)$. Again, this was deemed nonsignificant. In contrast, Nissen et al.'s $\mathrm{V}_{20 \mathrm{~Gy}} 70 \%$ reduction was significant (DIBH $2.3 \%$ vs. $7.8 \% \mathrm{FB}, p<0.0001)(15)$.

Results here, reflect Swanson, Hayden and Nissen et al.'s reports however were not significant. When examining the volumes receiving 20/30 Gy between the studies, there is a major division due to use of cardiac shielding (without compromising target coverage). Plans without cardiac shielding may experience increased dose reductions between
FB and DIBH. The statistical acceptance level used here, is also more conservative than the comparative studies.

LADCA irradiation, resulting from anatomical position, may aid risk determination of potential cardiac complications $(21,22)$. Left breast patients have increased cardiac complication risk compared to right (21-23). $70 \%$ of posttreatment cardiac abnormalities were localized in the LADCA by Correa et al. (21). Nilsson et al. established a direct link between left breast irradiation and increased LADCA stenosis, plus a relationship between high risk radiotherapy hotspots and coronary arterial stenosis (22). Therefore, LADCA dose consideration is imperative, to attempt reduction of cardiac complications.

Feng et al.'s cardiac contouring atlas was used to improve heart and LADCA delineation (9). However, Lorenzen et al. concluded that Feng's guidelines did not reduce variation/contouring errors (24). In response, the LADCAs were contoured separately to other organs-at-risk, case-bycase. They are difficult to contour and not always visible (e.g. at the heart base), hence guideline usage to reduce discrepancies. Exact LADCA dose-volume-constraints are difficult to establish at present, despite awareness of dose susceptibility. $\mathrm{D}_{10 \%}-\mathrm{D}_{100 \%}$, received significant reductions (Table VI), important given the LADCA's serial architecture, it cannot tolerate small volumes, irradiated to large doses causing functional compromise. This potentially could link to the coronary disease complications Correa et al. and Nilsson et al reported $(21,22)$.

LADCA $D_{\max }$ achieved a $47.83 \%$ reduction (mean $=15.56$ Gy, SD=10.62 Gy vs. FB mean=29.82 Gy, SD=10.05 Gy, $p<0.000)$. A $52 \%$ reduction in LADCA $\mathrm{D}_{\text {mean }}$ also resulted (mean=5.23 Gy, SD=1.94 Gy vs. FB mean=10.88 Gy, $\mathrm{SD}=3.95 \mathrm{~Gy}, p<0.000)$, Table II. These results are comparable with Vikstrom et al.'s $56.8 \% \mathrm{D}_{\max }$ reduction (38.7 Gy FB vs. 16.7 Gy DIBH) and $64.6 \% \mathrm{D}_{\text {mean }}$ reduction (18.1 Gy FB vs. 6.4 Gy DIBH) (25). In contrast, Anzar et al. established LADCA DIBH $D_{\text {mean }}=17.8$ Gy $\mathrm{SD}=14$ Gy contrasting with results here \& Taylor et al.'s $\mathrm{D}_{\text {mean }}=12.0 \mathrm{~Gy}, \mathrm{SD}=2.3 \mathrm{~Gy}$ for Swedish women treated in the 1990s using FB (26, 27). Anzar et al. suggests contouring and planning technique differences to explain the discrepancies, highlighting the importance of accurate LADCA contouring. Currently, LADCA dose complication risks are being researched in Scandinavia via the RACE study to establish potential dose volume constraints (28). LADCA dose may be a predictor of future coronary events and an important breast organ-at-risk.

Patients' amplitude level had no impact on dose reduction for OARs, Table VII. Amplitude also had no relationship with lung volume expansion and was not affected by smoking history. Only age and co-morbidity history had significant relationships with amplitude depths. This illustrates the simplicity of the external surrogate respiratory system, being heavily influenced by location and patients' 
chest motion. Patients' breathing cycle dictates motion, emphasizing the importance of documenting surrogate location for reproducibility. The external motion depth recorded is not relatable to internal motion expansion occurring. Elderly, co-morbid patients may generally have shallower breathing cycles and this is evident here. It is a major drawback regarding lack of quantifiable influence, compared to ABC (13). However, comparatively it is less invasive and more tolerable for patients (16). Importantly, DIBH capability is achievable allowing potential OAR dose reduction.

\section{Conclusion}

DIBH reduces dose to the heart and LADCA, without increased dose to the combined or ipsi-lateral lungs. Amplitude is an independent factor that is not correlated with DIBH dose benefits. The LADCA should be routinely contoured, for evaluation of dose impact and long term complications. LADCA dose was most affected by DIBH. Further evaluation and collaborative research is required to establish LADCA dose volume constraints. Dose reductions achieved using DIBH may potentially lead to reducing long term cardiac complications from left breast radiotherapy. Further research and long term follow-up is recommended to establish the clinical effects of DIBH.

\section{References}

1 International Agency for Research on Cancer. Breast Cancer Incidence \& Mortality Worldwide in 2008. World Health Organisation. Online available from: http://globocan.iarc.fr/ factsheets/cancers/breast.asp.

2 National Institute for Health and Care Excellence. Breast Cancer (Early \& Locally Advanced) Diagnosis and Treatment Guidelines. National Institute for Health and Care Excellence. Online available from: http://guidance.nice.org.uk/CG80.

3 Yang PS, Chen CM, Liu MC, Jian JM, Horng CF, Liu MJ, Yu BL, Lee MY and Chi CW: Radiotherapy can decrease locoregional recurrence and increase survival in mastectomy patients with $\mathrm{T} 1$ to $\mathrm{T} 2$ breast cancer and one to three positive nodes with negative estrogen receptor and positive lymphovascular invasion status.Int J Radiat Oncol Biol Phys 77(2): 516-522, 2009.

4 Clarke M, Collins R, Darby S, Davies C, Elphinstone P, Evans V, Godwin J, Gray R, Hicks C, James S, MacKinnon E, McGale P, McHugh T, Peto R, Taylor C, Wang Y; Early Breast Cancer Trialists' Collaborative Group (EBCTCG): Effects of radiotherapy and of differences in the extent of surgery for early breast cancer on local recurrence and 15-year survival: an overview of the randomised trials. Lancet 366(9503): 20872106, 2005.

5 Fisher B, Anderson S, Bryant J, Margolese RG, Deutsch M, Fisher ER, Jeong JH and Wolmark N: Twenty-year follow-up of a randomized trial comparing total mastectomy, lumpectomy, and lumpectomy plus irradiation for the treatment of invasive breast cancer. N Engl J Med 347(16): 1233-1241, 2002.
6 Early Breast Cancer Trialists' Collaborative Group: Favourable and unfavourable effects on long-term survival of radiotherapy for early breast cancer: an overview of the randomised trials. Lancet 355(9217): 1757-1770, 2000.

7 Marks LB, Yu X, Vujaskovic Z, Small W Jr, Folz R and Anscher MS: Radiation-induced lung injury. Semin Radiat Oncol 13(3): 333-345, 2003.

8 Gagliardi G, Bjöhle J, Lax I, Ottolenghi A, Eriksson F, Liedberg A, Lind P and Rutqvist LE: Radiation pneumonitis after breast cancer irradiation: analysis of the complication probability using the relative seriality model. Int J Radiat Oncol Biol Phys 46(2): 373-381, 2000.

9 Feng M, Moran JM, Koelling T, Chughtai A, Chan JL, Freedman L, Hayman JA, Jagsi R, Jolly S, Larouere J, Soriano J, Marsh R and Pierce LJ: Development and validation of a heart atlas to study cardiac exposure to radiation following treatment for breast cancer. Int J Radiat Oncol Biol Phys 79(1): 10-18, 2011.

10 Radiation Therapy Oncology Group: RTOG Breast Cancer Atlas, 2014. Online available from: http://www.rtog.org/ CoreLab/ ContouringAtlases/BreastCancerAtlas.aspx.

11 International Commission on Radiation Units and Measurements: ICRU Prescribing, Recording, and Reporting Photon Beam Therapy (Report 50). 1993 International Commission on Radiation Units and Measurements, Inc. Bethesda, MD, USA.

12 Prabhakar R, Tharmar G, Julka PK, Rath GK, Joshi RC, Bansal AK, Bisht RK, Gopishankar N, Pant GS and Thulkar S: Impact of different breathing conditions on the dose to surrounding normal structures in tangential field breast radiotherapy. J Med Phys 32(1): 24-28, 2007.

13 Swanson T, Grills IS, Ye H, Entwistle A, Teahan M, Letts N, Yan D, Duquette J and Vicini FA: Six-year experience routinely using moderate deep inspiration breath-hold for the reduction of cardiac dose in left-sided breast irradiation for patients with early-stage or locally advanced breast cancer. Am J Clin Oncol 36(1): 24-30, 2013.

14 Dincoglan F, Beyzadeoglu M, Sager O, Oysul K, Kahya YE, Gamsiz H, Uysal B, Demiral S, Dirican B and Surenkok S: Dosimetric evaluation of critical organs at risk in mastectomized left-sided breast cancer radiotherapy using breath-hold technique. Tumori 99(1): 76-82, 2013.

15 Nissen HD and Appelt AL: Improved heart, lung and target dose with deep inspiration breath hold in a large clinical series of breast cancer patients. Radiother Oncol 106(1): 28-32, 2012.

16 Hayden AJ, Rains M, Tiver K. Deep inspiration breath hold technique reduces heart dose from radiotherapy for left-sided breast cancer. J Med Imaging Radiat Oncol 56(4): 464-472, 2012.

17 Stranzl H and Zurl B: Postoperative irradiation of left-sided breast cancer patients and cardiac toxicity. Does deep inspiration breath-hold (DIBH) technique protect the heart? Strahlenther Onkol 184(7): 354-358, 2008.

18 Marks LB, Bentzen SM, Deasy JO, Kong FM, Bradley JD, Vogelius IS, El Naqa I, Hubbs JL, Lebesque JV, Timmerman RD, Martel MK and Jackson A: Radiation dose-volume effects in the lung. Int J Radiat Oncol Biol Phys 76(3 Suppl): S70-76, 2010.

19 Bruzzaniti V, Abate A, Pinnarò P, D'Andrea M, Infusino E, Landoni V, Soriani A, Giordano C, Ferraro A and Strigari L: Dosimetric and clinical advantages of deep inspiration breathhold (DIBH) during radiotherapy of breast cancer. 32(1): 88, 2013. 
20 Darby SC, Ewertz M, McGale P, Bennet AM, Blom-Goldman U, Brønnum D, Correa C, Cutter D, Gagliardi G, Gigante B, Jensen MB, Nisbet A, Peto R, Rahimi K, Taylor C and Hall P: Risk of ischemic heart disease in women after radiotherapy for breast cancer. N Engl J Med 368(11): 987-998, 2013.

21 Correa CR, Litt HI, Hwang WT, Ferrari VA, Solin LJ and Harris EE: Coronary artery findings after left-sided compared with right-sided radiation treatment for early-stage breast cancer. J Clin Oncol 25(21): 3031-3037, 2007.

22 Nilsson G, Holmberg L, Garmo H, Duvernoy O, Sjögren I, Lagerqvist B and Blomqvist C: Distribution of coronary artery stenosis after radiation for breast cancer. J Clin Oncol 30(4): 380-386, 2012.

23 Jagsi R, Griffith KA, Koelling T, Roberts R and Pierce LJ: Rates of myocardial infarction and coronary artery disease and risk factors in patients treated with radiation therapy for early-stage breast cancer. Cancer 109(4): 650-657, 2007.

24 Lorenzen EL, Taylor CW, Maraldo M, Nielsen MH, Offersen BV, Andersen MR, O'Dwyer D, Larsen L, Duxbury S, Jhitta B, Darby SC, Ewertz $\mathrm{M}$ and Brink C.: Inter-observer variation in delineation of the heart and left anterior descending coronary artery in radiotherapy for breast cancer: a multi-centre study from Denmark and the UK. Radiother Oncol 108(2): 254-258, 2013.
25 Vikström J, Hjelstuen MH, Mjaaland I and Dybvik KI: Cardiac and pulmonary dose reduction for tangentially irradiated breast cancer, utilizing deep inspiration breath-hold with audio-visual guidance, without compromising target coverage. Acta Oncol 50(1): 42-50, 2011.

26 Aznar MC, Korreman SS, Pedersen AN, Persson GF, Josipovic $\mathrm{M}$ and Specht L: Evaluation of dose to cardiac structures during breast irradiation. Br J Radiol 84(1004): 743-746, 2011.

27 Taylor CW, Nisbet A, McGale P, Goldman U, Darby SC, Hall P and Gagliardi G:. Cardiac doses from Swedish breast cancer radiotherapy since the 1950s. Radiother Oncol 90(1): 127-135, 2009.

28 Department of Medical Epidemiology and Biostatistics, Karolinska Institutet: Radiation Associated Cardiovascular Events, 2014. Online available from: http://www.race.ki.se/ index.html.

Received November 23, 2016

Revised January 24, 2017

Accepted January 26, 2017 